Cite this: J. Mater. Chem. C, 2014, 2, 5570

Received 14th April 2014 Accepted 28th May 2014

DOI: $10.1039 / c 4 t c 00744 a$

www.rsc.org/MaterialsC

\section{Air-stable photoconductive films formed from perylene bisimide gelators $\uparrow$}

\author{
Emily R. Draper, ${ }^{a}$ James J. Walsh, ${ }^{b}$ Tom O. McDonald, ${ }^{a}$ Martijn A. Zwijnenburg, ${ }^{c}$ \\ Petra J. Cameron, ${ }^{d}$ Alexander J. Cowan ${ }^{\star b}$ and Dave J. Adams ${ }^{\star a}$
}

\section{Introduction}

$\pi$-conjugated systems are used extensively in electronic devices, including solar cells and light emitting diodes. ${ }^{1}$ Such devices are normally formed using deposition or coating routes that can lead to the formation of aggregates with different size and shape distributions, which can influence the electronic properties. Self-assembly is a simple method of organizing optoelectronically active $\pi$-conjugated molecules in a defined manner with precise control at both the nano- and micro-scale. ${ }^{2-6}$ One selfassembly route is to exploit low molecular weight gelators (LMWG). ${ }^{2}$ Gelation using suitably designed LMWG results from self-assembly of the gelator into well-defined one-dimensional structures, which then can entangle or cross-link. When the LMWG contain $\pi$-conjugated groups, the result of this assembly is the stacking of these groups, which can be exploited to form conductive pathways for electronic devices. ${ }^{2}$ The assembly of LMWG based around perylene bisimides (PBI) is of great interest from the perspective of electronic materials, since PBIs are n-type materials. ${ }^{7-10}$ PBIs have strong absorption, long fluorescence lifetimes and high quantum yields. There are a growing number of reports showing the formation of selfassembled wires and fibers from PBI-based gelators. PBIs can also be (photo)conductive and have been suggested as candidates for many organic electronic devices; ${ }^{11-13}$ for example as

\footnotetext{
${ }^{a}$ Department of Chemistry, University of Liverpool, Crown Street, Liverpool, L69 7ZD, UK. E-mail: d.j.adams@liverpool.ac.uk

${ }^{b}$ Stephenson Institute for Renewable Energy, University of Liverpool, Peach Street, Liverpool, L69 7ZF, UK. E-mail: a.j.cowan@liverpool.ac.uk

'Department of Chemistry, University College London, 20 Gordon Street, London WC1H OAJ, UK

${ }^{d}$ Department of Chemistry, University of Bath, 1 South, Bath, BA2 7AY, UK

$\dagger$ Electronic supplementary information (ESI) available: The details of the synthesis, characterization data for all compounds, rheological data, addition photographs, UV-Vis and fluorescence data, SEMs, and conductivity data. See DOI: $10.1039 / \mathrm{c} 4 \mathrm{tc} 00744 \mathrm{a}$
}

alternative electron acceptors in solar cells ${ }^{7,14,15}$ or in field-effect transistors. ${ }^{16} \mathrm{PBIs}$ are readily sequentially reduced to the radical anion and dianion. Significant work has been done on the formation of one-dimensional structures based on PBIs, ${ }^{9,10,17,18}$ for example showing supramolecular chirality ${ }^{19}$ and the formation of liquid crystallinity. ${ }^{20} \mathrm{~A}$ key point is that the optoelectronic performance of such materials can depend not only on the chemical structure, but also on the morphology and uniformity of the aggregates formed. ${ }^{21,22}$ For PBIs, the aggregates formed can be controlled by functional group, solvent, or concentration. ${ }^{21,23}$ Exciton diffusion has been shown to occur in one-dimension in PBI-aggregates. ${ }^{24}$ The majority of the reported PBI-based LMWG gel organic solvents, due to the high hydrophobicity of the perylene group. Examples have been used in light harvesting and also in bulk heterojunctions, formed from self-sorted gel fibers. ${ }^{25}$ Recently, PBI-based hydrogelators have been reported, ${ }^{26}$ for example, an amino acid-based PBI was shown to form photoconductive xerogels. ${ }^{27}$ The LUMO level of an aspartic acid-based PBI has been suggested to be suitable for use as an electron acceptor in a solar cell; however the reduced PBI solution formed was highly sensitive to the presence of $\mathrm{O}_{2}$, which is not promising for OPV applications. It is therefore highly desirable to have reduced PBI species in thin films which are resistant to anion oxidation by $\mathrm{O}_{2}{ }^{28}$

\section{Results and discussion}

Here we discuss the photoconductivity of four PBIs LMWG, prepared by the reaction of 3,4:9,10-perylenetetracarboxyldianhydride with a number of amino acids following literature procedures for the coupling of amino acids to the anhydrides. ${ }^{28-30} 1$ and 2 (Fig. 1) have been previously reported ${ }^{29,30} \mathrm{We}$ focus on the L-alanine (1), L-histidine (2), L-phenylalanine (3) and L-valine (4) functionalized PBIs (Fig. 1).

1-4 all form deep red solutions at high $\mathrm{pH}(\approx \mathrm{pH} 10)$ at a concentration of $5 \mathrm{mg} \mathrm{mL}^{-1}$ (photographs are shown in Fig. 2a 


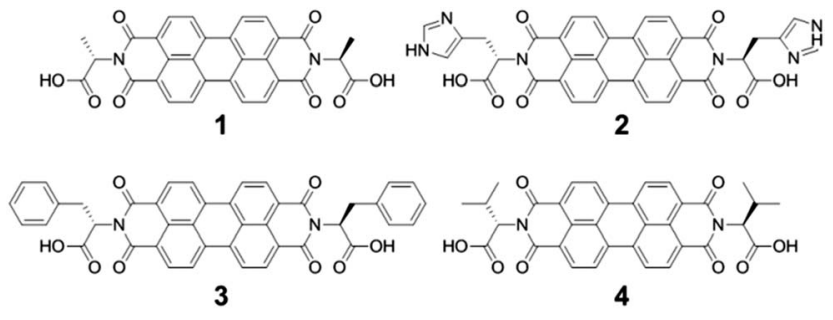

Fig. 1 Structures of the PBIs used in this study.

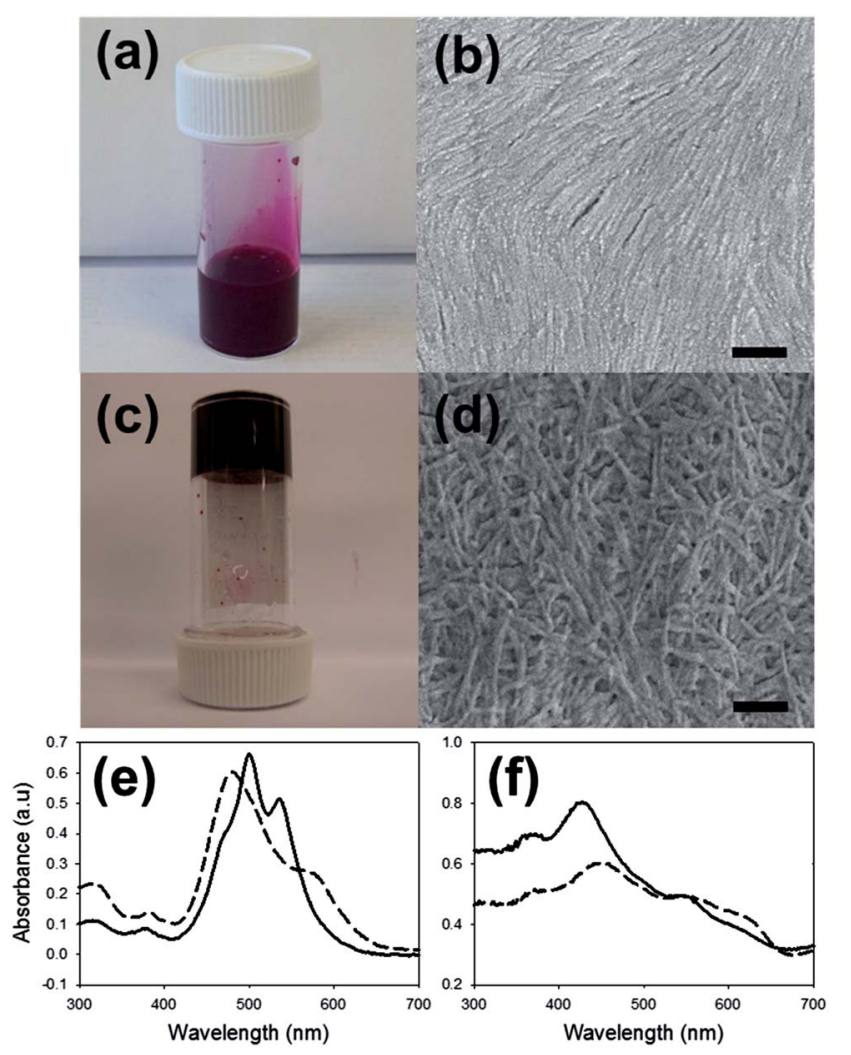

Fig. 2 (a) Photograph of a solution of 1 at $\mathrm{pH} 10$ at a concentration of 5 $\mathrm{mg} \mathrm{mL}^{-1}$. (b) SEM image of structures formed on drying the solution shown in (a). (c) A gel formed on acidification of the solution of 1 in (a). (d) SEM image of structures formed on drying the gel shown in (c). For (b) and (d), the scale bar represents $2 \mu \mathrm{m}$. (e) UV-Vis spectra of the solution (-) and gel (- - -). (f) UV-Vis spectra of the dried solution and the dried gel.

for 1; data for 2-4 are shown in Fig. S7-S9, ESI $\dagger$ ). Viscosity measurements of solutions of 1-4 at high $\mathrm{pH}$ show them to be shear thinning (Fig. S10†), suggesting that structures present are aligning at high shear causing the lower viscosity. Solutions of 3 have much higher viscosity than the other LMWG. We have previously noted that certain hydrophobic LMWG with similar molecular structures form worm-like micelles at high $\mathrm{pH},{ }^{31,32}$ leading to viscous solutions. Hence, it is likely that worm-like micelles are formed at high $\mathrm{pH}$ from 1-4. Indeed, the dried solutions generally showed the presence of long one-dimensional structures by SEM (Fig. $2 \mathrm{~b}$ and S7-S9†). Dried solutions of 3 however only showed disordered structures (Fig. S8 $\dagger$ ).
The viscous solutions formed self-supporting dark red gels on lowering the $\mathrm{pH}$ (again, photographs are shown in Fig. 2c for 1; data for 2-4 in Fig. S7-S9†). We adjusted the $\mathrm{pH}$ by adding glucono- $\delta$-lactone, which hydrolyses slowly to gluconic acid, ${ }^{33}$ resulting in a homogeneous $\mathrm{pH}$ drop for LMWG. ${ }^{34}$ On gelation, 1, 2, and 4 form transparent gels while $\mathbf{3}$ forms gels which are turbid. The rheological properties of these gels are similar for those formed from related $\mathrm{LMWG},{ }^{27}$ with the storage modulus $\left(G^{\prime}\right)$ and loss modulus $\left(G^{\prime \prime}\right)$ being only weakly dependent on frequency (Fig. S15-S18 $\dagger$ ). SEM of the dried gels (xerogels) showed the presence of thin entangled fibers. SEM of the dried solutions showed similar structures, with greater alignment of the fibrous structures than the corresponding xerogels. In all cases, powder X-ray diffraction (pXRD) data shows both the dried solutions and xerogels contain a low degree of crystallinity (Fig S11-S14 $\dagger$ ). The most intense peak in all samples is at $2 \theta=$ $\sim 25.5^{\circ}$, corresponding to approximately $3.5 \AA$, arising from $\pi-\pi$ stacking.

The absorption spectra of these materials in solution $(5 \mathrm{mg}$ $\mathrm{mL}^{-1}$ ) are typical of PBI-LMWG. UV-Vis data at high $\mathrm{pH}$ (Fig. 2e and $\mathrm{S} 20-\mathrm{S} 22 \dagger$ ) showed a shoulder at $470 \mathrm{~nm}$, and peaks at $490 \mathrm{~nm}$ and $540 \mathrm{~nm}$, attributed to the $0-0$ and $0-1$ vibronic bands of the $S_{0} \rightarrow S_{1}$ transitions. ${ }^{35}$ The ratio of the peak intensities at $490 \mathrm{~nm}$ and $540 \mathrm{~nm}$ indicates a significant degree of aggregation..$^{26,36}$ In the gel state, the UV-Vis data showed strong absorption at $470 \mathrm{~nm}$ and $590 \mathrm{~nm}$, with the peak at 470 nm now being dominant (Fig. 2e and S20-S22 $\dagger$ ). This change in relative intensity suggests a change in the aggregation of the perylenes. ${ }^{26}$ The spectra for both the solution and gel showed peaks at $325 \mathrm{~nm}$ and $380 \mathrm{~nm}$, corresponding to the electronic $\mathrm{S}_{0}$ $\rightarrow \mathrm{S}_{2}$ transition. $^{35}$ Fluorescence data showed fine structure emission with maxima at $540 \mathrm{~nm}$ and $595 \mathrm{~nm}$ for the solution on excitation at $365 \mathrm{~nm}$ or $490 \mathrm{~nm}$ (Fig. S22†). The fluorescence intensity of the gel is significantly lower due to self-quenching, with the peak maxima slightly shifted to shorter wavelength, again due to a change in aggregation.

PBIs are well known to be photoconductive, with a number of photoconductive LMWG systems having been reported. ${ }^{26,27,37}$ To investigate the photoconductivity of these materials, we dried both the solution and gel phases. Films were readily obtained from both the solutions and gels of 1, 2 and 4 simply by drying in air (final water content $\sim 6$ wt $\%$ by TGA). For 3 , drying led to an inhomogenous film that did not adhere well to the substrate (Fig. S23†), correlating with the SEM data. There are fundamental changes in the UV-Vis spectra on drying. For both dried solutions and xerogels, the UV-Vis data showed an increase of absorbance in the UV region where there is a change in the intensity ratio of the peaks at $375 \mathrm{~nm}$ and those in the region of $470-590 \mathrm{~nm}$. This suggests a difference in the arrangement of the perylene aggregates upon drying. The macroscopic conductivity of the dried solutions and gels was measured both in the dark and under illumination with a xenon lamp. Both showed Ohmic response and a significantly increased current under illumination, assigned to the samples becoming photoconductive (see Fig. S24-27†), with symmetric data during the voltage sweep. In general, the dried solutions were more conductive than the xerogels. 1 showed the highest 
current under illumination of the LMWG, and the photoresponse was significantly greater for the dried solution than for the dried gel. 3 showed a very weak response, which we attribute primarily to the poor film quality.

However, in all cases, despite the expected correlation between the absorption spectrum of the perylene group and the proposed photoconductivity, both the dried gel and solution remain highly resistive when irradiated with light above 400 $\mathrm{nm}$. Instead, a significant photocurrent was only induced when irradiated with wavelengths shorter than $400 \mathrm{~nm}$ (Fig. 3a and S28-S30 $)$. The onset wavelength of the photoresponse of the dried solution and xerogel varied. For 1, 2 and 4, the xerogel resistance decreased significantly with irradiation of wavelengths shorter than $400 \mathrm{~nm}$, whereas the dried solutions only became active when irradiated at wavelengths of $375 \mathrm{~nm}$ or shorter. Due to the poor film quality, we did not attempt to collect this data for 3 . Irradiation of samples of 1, 2 and 4 with $365 \mathrm{~nm}$ LED irradiation again showed a significant decrease in resistance, indicating photoconductivity. For $\mathbf{1}$ and $\mathbf{4}$, the dried solution was found to be approximately one order of magnitude less resistive under irradiation than the xerogel (Fig. 3b). This may be due to the packing in the solution versus gel state, but could also be due to differences in fiber thicknesses, density, and orientation. However, the photocurrents measured in our two electrode experiment were consistently greater with both the dried solutions and xerogels of 1 than for $\mathbf{2}$ and $\mathbf{4}$, indicating a greater degree of photoconductivity. The conductivity for PBIs has been related to well-ordered $\pi$-stacking and the morphology has also been shown to be key. ${ }^{21}$ The UV-Vis and pXRD data for 1, 2 and $\mathbf{4}$ are similar; hence, we ascribe the higher conductivity of 1 to differences in fiber morphology over molecular packing.

Focusing on 1, on irradiating a dried solution in a two electrode cell $(2 \mathrm{~V})$ with $365 \mathrm{~nm}$ light, the photocurrent
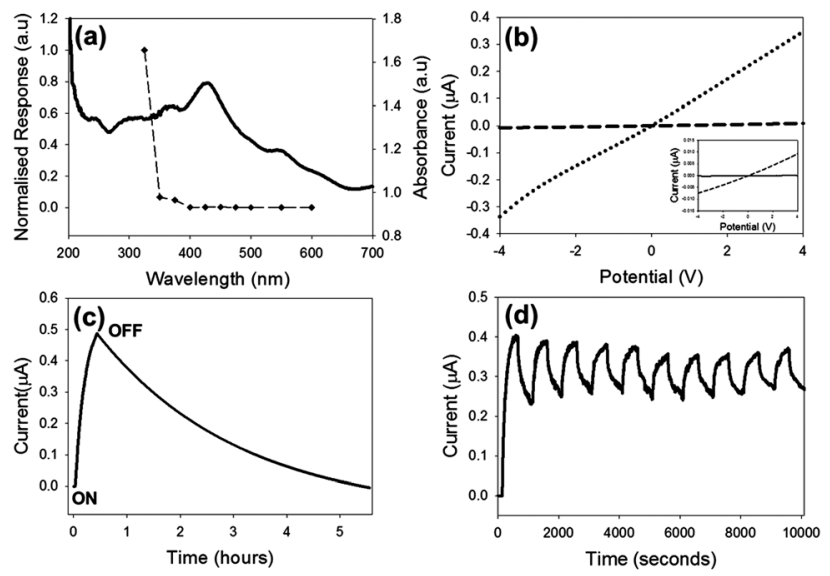

Fig. 3 (a) Plot of photocurrent (at $2 \mathrm{~V}$ potential difference) against irradiation wavelength for a dried solution of 1 overlaid with the UV-Vis spectrum. (b) Plots of $I-V$ curves for $(\cdots)$ a dried solution of 1 and (- - -) a xerogel for 1 on irradiation at $365 \mathrm{~nm}$. Inset is the data for the xerogel (- - -) under irradiation and (-) in the dark. (c) Photoresponse for a xerogel of 1, initially in the dark, then irradiated with $365 \mathrm{~nm}$ light, followed by the lamp being turned off. (d) Transient photoresponse for a dried solution of 1 by turning on and off the $365 \mathrm{~nm}$ light for multiple cycles. increases with time (Fig. 3c). On switching off the light, photocurrent persists for typically 1-8 hours, with the decay shown in Fig. 3c being fitted to a single exponential process, with a lifetime of $c a .5300$ s. This indicates that the photoinduced conductivity is remarkably long-lived in air. There is variability from sample to sample, due to differences in film thicknesses and fiber density and orientation, but the films show lifetimes consistently $>3000 \mathrm{~s}$. Switching the light on and off demonstrated the stability of the films (Fig. 3d). Similar behavior was observed for the xerogel (Fig. S31†).

On irradiation with $365 \mathrm{~nm}$ light, both the xerogel (Fig. 4a) and dried solution (Fig. 4b) were also observed to change color. This color change was reversible over several hours. Monitoring this process by UV-Vis-NIR spectroscopy for the xerogel, we observed the formation of a new absorption feature with maxima at $735 \mathrm{~nm}, 820 \mathrm{~nm}$ and $1000 \mathrm{~nm}$ (Fig. 4c), in good agreement with data for the formation of the radical anion ${ }^{38}$ formed by chemical reduction. For the dried solution, similar absorptions were observed, albeit with a higher relative intensity, in addition to an increase in the relative intensity of the shoulder at $615 \mathrm{~nm}$ (Fig. 4d), as expected for the dianion. ${ }^{28} \mathrm{We}$ confirmed that these peaks were characteristic of these species
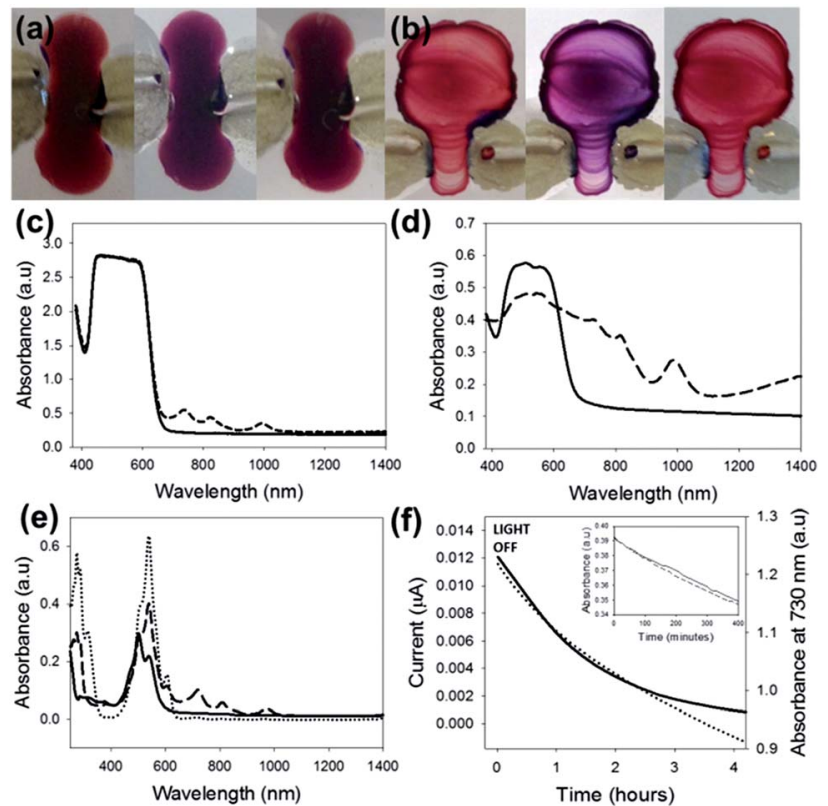

Fig. 4 (a) On irradiation with $365 \mathrm{~nm}$ light, a xerogel of 1 (left) changes color (middle). After the light is turned off, the original color slowly returns (right), photograph after 18 hours. (b) A similar effect is observed for the dried solution. (c) UV-Vis-NIR spectra for a xerogel of $1(-)$, and for the same film 1 minute after irradiation with $365 \mathrm{~nm}$ light (- - -). (d) UV-Vis-NIR spectra for a dried solution of 1 (-), and for the same film 1 minute after irradiation with $365 \mathrm{~nm}$ light (- - -). (e) UV-VisNIR showing absorbance of 1 in solution after addition of various amounts of sodium dithionite. Solid line represents before sodium dithionite was added, dashed line is after $20 \mathrm{mg}$ of sodium dithionite was added (radical anion of 1) and the dotted line is after $40 \mathrm{mg}$ of sodium dithionite was added (dianion). (f) Comparison of rate of decrease in conductivity and absorbance at $730 \mathrm{~nm}$. Inset: change of absorbance at $730 \mathrm{~nm}$ with time after the $365 \mathrm{~nm}$ LED was turned off in air (- - ) and under argon (-). 
by reduction of $\mathbf{1}$ in solution using sodium dithionite (Fig. $4 \mathrm{e}$ and S38†). ${ }^{39}$ On the basis of these data, we suggest that the higher anion concentration in the dried solution explains the higher conductivity of the dried film over the dried gel. The possible presence of the dianion in the dried solution may also contribute, but the overlap of the spectral features with the ground state and anion prevent definitive assignment. We again highlight however that this apparent higher conductivity could also be due to differences in the density of the film, orientation of fibres or fibre thickness (see above). Simultaneously measuring the UV-Vis spectrum and photocurrent after switching off the LED showed that the initial decay was similar (Fig. 4f), and that the conductivity was essentially zero at the point where approximately $20 \%$ of the intensity for the peak at $730 \mathrm{~nm}$ remained. This is unsurprising; for conductivity a contiguous percolation pathway is required, and hence bulk conductivity is expected to decay before the presence of any conductive species.

The insensitivity of the spectral features of the decay to $\mathrm{O}_{2}$ was further demonstrated by comparing the decay in air compared to that under argon. The rate of decay was very similar in both cases (Fig. 4f, inset). Importantly, no changes in the UV-Vis spectra were observed when the samples were irra-

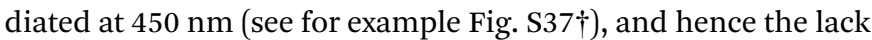
of photocurrent when irradiated at higher wavelengths can be ascribed to the lack of formation of either radical anion or dianion. The correlation between conductivity and the presence of the radical anion is expected. For example, a thin film of a PBI has been shown to form the radical anion by a self-doping mechanism on dehydration in air. ${ }^{15}$ A concomitant increase in conductivity was observed. What are perhaps surprising is both the wavelength dependence of the conductivity found here and the $\mathrm{O}_{2}$ tolerance of the photoconductivity. The lowest energy required for light to be absorbed in a material is referred to as the optical gap. Absorption of light of this energy results in the formation of excitons; excited electron-hole pairs bound through electrostatic interaction. Photoconductivity, however, requires not neutral excitons but free charge carriers (free electrons, free holes, or both), where the label free signifies that the charge carriers are not bound together in an exciton and hence able to contribute to electronic conductivity. The minimum energy needed to generate such free charge carriers is the transport (or quasiparticle) gap. This transport gap is always larger or equal to the optical gap, with the difference between the two equal to the exciton binding energy $E_{\text {ebe }}$; the amount of energy by which excitons are stabilized with respect to free electrons and holes. $E_{\text {ebe }}$ can be very small, e.g. in elemental and compound semiconductors, but for organic materials is typically in the order of tenths of an eV. Hence, we ascribe the difference between absorption and photoconductivity onset to the $E_{\text {ebe }}$, the extra energy required to form free electrons and holes from excitons.

An enhancement in conductivity for a PBI has been found previously on generation of the radical anion using hydrazine vapor. ${ }^{40}$ Elsewhere, the conductivity of PBI fibers was increased in the presence of diethylamine. ${ }^{26}$ In both these cases, a strong electron donor was present. In our work, no such donor is present, explaining the greater $E_{\text {ebe }}$ here. The fate of the cation is not clear. Whilst there is significant data available on the radical anion and dianion for a range of PBIs, there is less on the radical cation. Data has been reported for bay-substituted PBIs, ${ }^{41-43}$ with radical cations absorbing in the near-IR (NIR) region, however direct comparison to our materials is difficult. We do observe a broad peak above $1200 \mathrm{~nm}$ in the UV-Vis spectrum for the dried solution of $\mathbf{1}$ after illumination with $365 \mathrm{~nm}$ light (Fig. 4d) that on the basis of the solution phase chemical reduction experiment is not assigned to the radical anion or dianion (Fig. 4e). This feature grows in under UV illumination with a maximum at $\sim 1700 \mathrm{~nm}$, and decays at the same rate as the radical anion. It is possible that the NIR absorption is due to the radical cation, although this would represent a remarkably long-lived charge separated state. Alternatively, it may be assignable to a morphological change following irradiation, and further studies are ongoing.

The slow rate of decay of the photocurrent is surprising. The $\mathrm{PBI}$ radical anion in solution can be used as a sensitive probe for $\mathrm{O}_{2}{ }^{28,44}$ Our materials behave similarly (Fig. S38 $\dagger$ ). However, when dried as a film, it is clear that the $\mathrm{O}_{2}$ sensitivity is significantly reduced. Indeed, all of the data above are for measurements carried out in air. Under an argon atmosphere, the rate of decrease was similar to that in air; this shows that $\mathrm{O}_{2}$ has little effect on the recovery of the dried sample (Fig. 4f). An issue with many n-type semiconductors is the lack of stability in air. For example, photoconductive PBI-based nanofibers have been shown to be highly sensitive to the presence of $\mathrm{O}_{2},{ }^{37}$ where the photoconductivity was found to be three times higher under argon than under air. This was ascribed to high surface area available due to the morphology and the scavenging ability of $\mathrm{O}_{2}$. However, in other cases, the morphology can result in a kinetic barrier to the intrusion of water or $\mathrm{O}_{2}$, for example by using PBIs containing perfluorinated substituents. ${ }^{45,46}$ It has also been reported that aggregation stabilizes the radical anion. When incorporated in a film, the stability of the radical anion to oxygen was found to be higher, taking 20 minutes to be re-oxidized, as opposed to being immediately re-oxidized when in homogeneous solution. ${ }^{47}$ This was attributed to slow diffusion of $\mathrm{O}_{2}$ into the film. The slow rate of decay in our systems is significantly longer than for this system. ${ }^{47}$ It is unclear as to whether this is due to film thickness, morphology, or a result of the decreased diffusion rates of $\mathrm{O}_{2}$ through the films.

\section{Conclusions}

In conclusion, PBIs are of increasing interest for applications in organic electronics, including as alternative acceptor materials in OPV. ${ }^{7}$ Morphological control of such materials is likely to be critical for controlling their use. Here, we report self-assembling PBIs, which form long $1 \mathrm{D}$ structures that are photoconductive. The conductivity correlates with the presence of the radical anion, which is found to be remarkably long lived (on a timescale of hours), even in air and in the presence of water. The different molecules use here show different behaviour. For LMWG, small changes in molecular structure can have profound effects on the self-assembly and this is again 
exemplified here for 1-4. It is clear from the SEM images that the more ordered structures formed by 1 lead to higher photoconductivity; the reasons why $\mathbf{1}$ forms such structures as compared to 2-4 is currently under investigation. The materials presented here show markedly different conduction properties to those in the literature. Typically, the photoconductivity decays very rapidly (sub second timescales), and the decay is further accelerated by $\mathrm{O}_{2}$. The materials presented here maintain their photoconductivity for several hours after the light is turned off due to the high stability of the radical anion. It should also be noted that the gels and solutions presented here contain well defined 1D structures, which will be invaluable in a wide range of applications.

\section{Acknowledgements}

We thank the EPSRC for a DTA (ERD). We thank Prof. K. Durose and Dr R. Treharne (SIRE) for providing access to the UV-VisNIR spectrometer. MAZ and AJC acknowledge the EPSRC for Fellowships (EP/I004424/1) and (EP/K006851/1) respectively.

\section{Notes and references}

1 A. Facchetti, Chem. Mater., 2010, 23, 733-758.

2 S. S. Babu, V. K. Praveen and A. Ajayaghosh, Chem. Rev., 2014, 114, 1973-2129.

3 G. Koshkakaryan, P. Jiang, V. Altoe, D. Cao, L. M. Klivansky, Y. Zhang, S. Chung, A. Katan, F. Martin, M. Salmeron, B. Ma, S. Aloni and Y. Liu, Chem. Commun., 2010, 46, 8579-8581.

4 L. M. Klivansky, D. Hanifi, G. Koshkakaryan, D. R. Holycross, E. K. Gorski, Q. Wu, M. Chai and Y. Liu, Chem. Sci., 2012, 3, 2009-2014.

5 J. Zhao, J. I. Wong, J. Gao, G. Li, G. Xing, H. Zhang, T. C. Sum, H. Y. Yang, Y. Zhao, S. L. Ake Kjelleberg, W. Huang, S. C. Joachim Loo and Q. Zhang, RSC Adv., 2014, 4, 1782217831.

6 J. Zhao, J. I. Wong, C. Wang, J. Gao, V. Z. Y. Ng, H. Y. Yang, S. C. J. Loo and Q. Zhang, Chem. - Asian J., 2013, 8, 665-669.

7 C. Li and H. Wonneberger, Adv. Mater., 2012, 24, 613-636.

8 S. Yagai, M. Usui, T. Seki, H. Murayama, Y. Kikkawa, S. Uemura, T. Karatsu, A. Kitamura, A. Asano and S. Seki, J. Am. Chem. Soc., 2012, 134, 7983-7994.

9 D. Görl, X. Zhang and F. Würthner, Angew. Chem., Int. Ed., 2012, 51, 6328-6348.

10 F. Wurthner, Chem. Commun., 2004, 1564-1579.

11 Y. Che, H. Huang, M. Xu, C. Zhang, B. R. Bunes, X. Yang and L. Zang, J. Am. Chem. Soc., 2010, 133, 1087-1091.

12 C. Huang, S. Barlow and S. R. Marder, J. Org. Chem., 2011, 76, 2386-2407.

13 C. Huang, M. M. Sartin, N. Siegel, M. Cozzuol, Y. Zhang, J. M. Hales, S. Barlow, J. W. Perry and S. R. Marder, J. Mater. Chem., 2011, 21, 16119-16128.

14 W. Jiang, L. Ye, X. Li, C. Xiao, F. Tan, W. Zhao, J. Hou and Z. Wang, Chem. Commun., 2014, 50, 1024-1026.

15 T. H. Reilly, A. W. Hains, H.-Y. Chen and B. A. Gregg, Adv. Energy Mater., 2012, 2, 455-460.
16 J. M. Mativetsky, E. Orgiu, I. Lieberwirth, W. Pisula and P. Samorì, Adv. Mater., 2014, 26, 430-435.

17 J. K. Gallaher, E. J. Aitken, R. A. Keyzers and J. M. Hodgkiss, Chem. Commun., 2012, 48, 7961-7963.

18 L. Tian, R. Szilluweit, R. Marty, L. Bertschi, M. Zerson, E.-C. Spitzner, R. Magerle and H. Frauenrath, Chem. Sci., 2012, 3, 1512-1521.

19 P. K. Sukul, P. K. Singh, S. K. Maji and S. Malik, J. Mater. Chem. B, 2013, 1, 153-156.

20 R. A. Cormier and B. A. Gregg, J. Phys. Chem. B, 1997, 101, 11004-11006.

21 Y. Sun, C. He, K. Sun, Y. Li, H. Dong, Z. Wang and Z. Li, Langmuir, 2011, 27, 11364-11371.

22 Y. Chen, Y. Feng, J. Gao and M. Bouvet, J. Colloid Interface Sci., 2012, 368, 387-394.

23 K. Balakrishnan, A. Datar, T. Naddo, J. Huang, R. Oitker, M. Yen, J. Zhao and L. Zang, J. Am. Chem. Soc., 2006, 128, 7390-7398.

24 H. Marciniak, X.-Q. Li, F. Würthner and S. Lochbrunner, J. Phys. Chem. A, 2010, 115, 648-654.

25 K. Sugiyasu, S.-i. Kawano, N. Fujita and S. Shinkai, Chem. Mater., 2008, 20, 2863-2865.

26 A. Datar, K. Balakrishnan and L. Zang, Chem. Commun., 2013, 49, 6894-6896.

27 S. Roy, D. Kumar Maiti, S. Panigrahi, D. Basak and A. Banerjee, RSC Adv., 2012, 2, 11053-11060.

28 L. Zhong, F. Xing, W. Shi, L. Yan, L. Xie and S. Zhu, ACS Appl. Mater. Interfaces, 2013, 5, 3401-3407.

29 W. Tuntiwechapikul, T. Taka, M. Béthencourt, L. Makonkawkeyoon and T. Randall Lee, Bioorg. Med. Chem. Lett., 2006, 16, 4120-4126.

30 Y. Xu, S. Leng, C. Xue, R. Sun, J. Pan, J. Ford and S. Jin, Angew. Chem., Int. Ed., 2007, 46, 3896-3899.

31 L. Chen, G. Pont, K. Morris, G. Lotze, A. Squires, L. C. Serpell and D. J. Adams, Chem. Commun., 2011, 47, 12071-12073.

32 L. Chen, T. O. McDonald and D. J. Adams, RSC Adv., 2013, 3, 8714-8720.

33 Y. Pocker and E. Green, J. Am. Chem. Soc., 1973, 95, 113119.

34 D. J. Adams, M. F. Butler, W. J. Frith, M. Kirkland, L. Mullen and P. Sanderson, Soft Matter, 2009, 5, 1856-1862.

35 S. Akimoto, A. Ohmori and I. Yamazaki, J. Phys. Chem. B, 1997, 101, 3753-3758.

36 C. Backes, C. D. Schmidt, K. Rosenlehner, F. Hauke, J. N. Coleman and A. Hirsch, Adv. Mater., 2010, 22, 788802.

37 Y. Che, X. Yang, G. Liu, C. Yu, H. Ji, J. Zuo, J. Zhao and L. Zang, J. Am. Chem. Soc., 2010, 132, 5743-5750.

38 R. O. Marcon and S. Brochsztain, J. Phys. Chem. A, 2009, 113, 1747-1752.

39 E. Shirman, A. Ustinov, N. Ben-Shitrit, H. Weissman, M. A. Iron, R. Cohen and B. Rybtchinski, J. Phys. Chem. B, 2008, 112, 8855-8858.

40 Y. Che, A. Datar, X. Yang, T. Naddo, J. Zhao and L. Zang, J. Am. Chem. Soc., 2007, 129, 6354-6355.

41 M. J. Ahrens, M. J. Tauber and M. R. Wasielewski, J. Org. Chem., 2006, 71, 2107-2114. 
42 A. S. Lukas, Y. Zhao, S. E. Miller and M. R. Wasielewski, J. Phys. Chem. B, 2002, 106, 1299-1306.

43 M. Berberich, A.-M. Krause, M. Orlandi, F. Scandola and F. Würthner, Angew. Chem., Int. Ed., 2008, 47, 66166619.

44 I.-S. Shin, T. Hirsch, B. Ehrl, D.-H. Jang, O. S. Wolfbeis and J.-I. Hong, Anal. Chem., 2012, 84, 9163-9168.
45 B. A. Jones, M. J. Ahrens, M.-H. Yoon, A. Facchetti, T. J. Marks and M. R. Wasielewski, Angew. Chem., Int. Ed., 2004, 43, 6363-6366.

46 J. H. Oh, S. Liu, Z. Bao, R. Schmidt and F. Würthner, Appl. Phys. Lett., 2007, 91, 212107.

47 R. O. Marcon and S. Brochsztain, Langmuir, 2007, 23, 1197211976. 